\title{
OBITUARI: ÀNGEL ANGLADA (1927-2020)
}

\author{
XAVIER ESPLUGA \\ Universitat de Barcelona \\ xespluga@ub.edu \\ ORCID: 0000-0002-6690-1974
}

El proppassat 2 de març de 2020, al llindar dels 93 anys, traspassava a Sant Cugat, on residia des de feia gairebé vint anys, el doctor Àngel Anglada Anfruns (Barcelona, 25.3.1927 - Sant Cugat del Vallès, 2.3.2020) que fou professor de Filologia Llatina a la Universitat de Barcelona, a la Universitat Autònoma de Barcelona i a la Universitat de València.

Anglada havia nascut en el si d'una família benestant i molt catòlica, d'arrels vigatanes, bagenques i berguedanes. Fou el cinquè i darrer fill del matrimoni format per Àngel Maria Anglada Fuxà i Maria del Queralt Anfruns Ribas († 1987) (havent estat precedit per Antoni, Mercè, Montserrat i Josep Maria). Per la branca paterna pertanyia a una nissaga de metges odontòlegs, iniciada pel seu avi Antoni Anglada Torras (Vic 1850 - Barcelona 1919), considerat un dels pioners de l'odontologia catalana, ${ }^{1} \mathrm{i}$ continuada pel seu pare, el seu germà Josep Maria († 2007) i els seus nebots. El seu avi matern, Josep Anfruns i Espelt, també s'havia dedicat a la medicina. ${ }^{2}$ Foren metges militars tant un oncle de la banda materna, Mariano Anfruns Armengol (també oncle i padrí del periodista Carles Sentís Anfruns, cosí d'Anglada), com el seu oncle patern Ramon Anglada Fuxà.

Amb el precedent familiar que suposava el seu germà gran Antoni († 1990), sacerdot i prevere, ${ }^{3}$ també el jove Àngel Anglada va abraçar la condició religiosa. Estudià al Seminari Conciliar de Barcelona i fou ordenat sacerdot el 4 de març del 1951; no abandonaria els hàbits fins a finals dels 60; però, fins i tot després de la seva secularització, per la força del costum, molts dels qui l'havien vist donar classes abillat amb la sotana continuaren anomenant-lo "padre Anglada". En els anys de Seminari, es va despertar en Anglada l'interès per dues de les temàtiques que abordaria en el futur: la transmissió textual -és seu el llistat de manuscrits conservats a l'Arxiu de la Catedral publicat com a apèndix a la conferència inaugural del curs acadèmic pronunciada per Josep Oliveras Caminal ${ }^{4}$ - i la figura de Pacià, bisbe de Barcelona (des del 365/373 dC), a proposta del mateix Oliveras, que aleshores era el canonge arxiver de la Catedral de Barcelona.

\footnotetext{
1 Vegeu-ne la semblança a la Galeria de metges de Catalunya disponible a http://www.galeriametges.cat/galeria-fitxa.php?icod=EFFD.

${ }_{2}^{2}$ Per l'evocació dels Anfruns, vegeu SenTís (2006), 21, 24-25.

${ }^{3}$ Antoni Anglada es llicencià en Dret per la Universitat de Barcelona l'any 1936. Fou ordenat sacerdot el 1941.

${ }^{4}$ Oliveras Caminal (1949). Vegeu també Oliveras Caminal (1952).
} 
Anglada es llicencià en Filosofia i Lletres (1951-1956), en l'especialitat de Filologia Clàssica, a la Universitat de Barcelona i cursà els estudis de doctorat a la mateixa institució. La seva tesi doctoral (1964) -centrada, com veurem, en la figura de Pacià de Barcelona $^{5}$ - fou dirigida per Lisardo Rubio (1915-2006), que aleshores era Catedràtic de Llengua i Literatura llatina de la UB (1950-1967). Anglada complementà la formació rebuda a Barcelona amb freqüents estades a l'estranger, especialment a Alemanya, on entrà en contacte amb Klaus Sallmann i Antonie Wlosok († 2013), professors a la Universitat de Magúncia, i amb Wilfried Stroh, que li deixava el seu pis de Munic mentre era de vacances. La biblioteca instrumental que havia anat acumulant amb el pas del temps li va permetre de suplir les tradicionals mancances bibliogràfiques dels nostres centres universitaris.

Des del punt de vista professional, Anglada va guanyar una càtedra de "Llatí" d'Ensenyament Mitjà l'any $1967^{6} \mathrm{i}$, tot seguit, va exercir com a professor de secundària a la delegació de Cornellà de l'INEM “Jaume Balmes", a l'INEM "Jacint Verdaguer" de Barcelona i a l'INEM de Santa Coloma de Gramenet (anomenat "Puig Castellar" des del 1971). ${ }^{7}$ També a partir d'aquell moment resseguí les diverses etapes del cursus acadèmic a la seva alma mater: fou ajudant de Rubio -en assignatures com ara els textos de cinquè curs (Tàcit)- i professor encarregat de curs de Filologia Llatina (1967-1972) -on, entre altres assignatures, va impartir "Literatura llatina cristiana"; posteriorment esdevingué professor agregat de Filologia Llatina de la UAB (1971-1973), ${ }^{8}$ professor agregat de Literatura Llatina de nou a la UB (1973-1978), ${ }^{9}$ catedràtic de Llengua i Literatura Llatines de la Universitat de València (1978-1992), ${ }^{10}$ i finalment professor emèrit d'aquest últim centre (1995-1997).

A l'Estudi General de la capital valenciana fou també director del Departament de Filologia Clàssica i l'encarregat de pronunciar el discurs d'apertura del curs acadèmic 1982/1983 amb una dissertació sobre l'edició de l'obra de Pacià que havia dut a terme l'any 1780 l'aristòcrata valencià Vicent Noguera Ramon (1728-1797), regidor de la Ciutat de València. ${ }^{11}$

Des del punt de vista de l'activitat investigadora, la figura de Pacià obre i tanca la carrera d'Anglada. De fet, la figura del prelat barceloní ja havia estat estudiada pel seu mestre, Lisardo Rubio, que, durant la seva estada a la UB, havia analitzat les fonts de Pacià, ${ }^{12}$ publicat una traducció castellana, apareguda a l'efímera Colección de Autores

\footnotetext{
5 Anglada (1964a).

${ }^{6}$ Per al nomenament, vegeu $B O E, 17.11 .1967$.

${ }^{7}$ El director del centre, entre 1968 i 1972, i responsable també del canvi de nom de l'INEM, fou el també llicenciat en Filologia Clàssica i estudiós d'Aristòtil, Joan Vaqué Jordi († 2018).

8 Per al nomenament, vegeu BOE 5.7.1971.

9 Per al nomenament, vegeu $B O E, 7.12 .1973$.

${ }_{10}$ Per al nomenament, vegeu $B O E, 21.10 .1978$

11 Anglada (1982).

12 Rubio (1957).
} 
Barceloneses que havia impulsat Agustí Duran i Sempere ${ }^{13}$ i dirigit diverses memòries de llicenciatura sobre l'obra pacianenca. ${ }^{14}$

La tesi doctoral d'Anglada, defensada a la UB l'any $1964,{ }^{15}$ va merèixer el premi a les tesis doctorals de la SEEC d'aquell any. Estava dedicada a la relació de Pacià amb els tres testimonis del sintagma perdut d'Hipòlit titulat "Contra totes les heretgies". Bona part de la seva argumentació fou publicada com a article al número de la prestigiosa revista Emerita de l'any següent. ${ }^{16}$ Com que la intenció d'Anglada era fer una edició crítica de la totalitat de l'obra, el filòleg barceloní va començar a interessar-se per la tradició textual, a examinar els principals testimonis manuscrits i a valorar-ne les edicions crítiques pretèrites. Anglada dedicà moltes de les seves contribucions escrites, aparegudes en les principals revistes catalanes (Faventia, Estudis Universitaris Catalans, Arxiu de Textos Catalans Antics), espanyoles (Emerita, Cuadernos de Filología Clásica, Helmantica, Analecta Malacitana) i internacionals (Vetera Christianorum), a l'obra de Pacià. També participà en moltes reunions científiques, en particular, els congressos d'estudis clàssics de la SEEC i els simposis de la Secció Catalana, tot i que un fet imprevist va impedir finalment la seva participació en el col-loqui internacional celebrat a Barcelona i a Lió l'any 1996, dedicat monogràficament a la figura del bisbe barceloní. ${ }^{17}$ En totes aquestes contribucions escrites -moltes de les quals foren recollides en la Silua studiorum, un volum antològic publicat per Brepols l'any 2012-18, Anglada hi vessà les seves preocupacions sobre el textus receptus de $1^{\prime}$ obra de Pacià, ${ }^{19}$ amb especial atenció a la tradició manuscrita, ${ }^{20}$ a les edicions i anotacions de l'obra pacianenca, ${ }^{21}$ a les fonts (en particular, a les característiques del text bíblic citat pel prelat), a la influència dels autors clàssics, sobretot de Virgili, ${ }^{22}$ al problema de la filiació genèrica d'algunes de les obres pacianenques, ${ }^{23} \mathrm{i}$ a qüestions sintàctiques i estilístiques (inclòs el fenomen de la prosa rítmica). ${ }^{24}$ També destacaria, per la profunditat, les dues recensions ${ }^{25}$ que Anglada va fer sobre el volum dedicat als Écrits de Pacià de Barcelona editat pel teòleg Carmelo Granado, S.I., († 2013) a la col-lecció Sources Chrétiennes (amb traducció francesa de Chantal Épitalon i Michel Lestienne). ${ }^{26}$ En qualsevol cas, els seus treballs sobre Pacià culminaren l'any $2012 \mathrm{amb}$ l'aparició de l'edició crítica de tota l'obra del prelat barceloní en el volum 69B de la Series

\footnotetext{
${ }^{13}$ Rubio (1958).

${ }^{14}$ García Gil (1960); Requena Gallego (1960).

15 Anglada (1964a).

${ }^{16}$ Anglada (1965).

17 Per a les actes del col-loqui, vegeu Bertrand, Busquets, Mayer (2004).

18 Anglada (2012a).

${ }^{19}$ Anglada (1979); Anglada (1980a); Anglada (1980b); Anglada (1982); Anglada (1983b); Anglada (1983d); Anglada (1984a); Anglada (1986b); Anglada (1988a); Anglada (1988b); Anglada (1991b);

20 Anglada (1998); Anglada (2006).

21 Anglada (1982); Anglada (1984b); Anglada (1986a); Anglada (1986c); Anglada (1991b); Anglada (1997a)

22 Anglada (1983d); Anglada (1985);

23 Anglada (1989b). Per a aquesta qüestió, vegeu també Tovar Paz (1994).

24 Anglada (1986-1987); Anglada (1989a); Anglada (1994).

25 Anglada (1997b); Anglada (1999);

${ }^{26}$ Granado (1995).
} 
Latina del Corpus Christianorum, ${ }^{27}$ que va dedicar a la seva esposa María del Carmen Postigo. Em consta també que Anglada ha deixat inèdita una traducció catalana -en la que feia temps que hi estava treballant ${ }^{28}$ - que esperem que pugui ser publicada amb dignitat en temps no gaire llunyans. ${ }^{29}$

El text de Pacià portà Anglada a abordar altres qüestions. Com que un dels principals manuscrits de la seva obra -l'actual ms. Vat. Reg. Lat. 331- conservava restes de la puntuació antiga, es va començar a interessar pels estudis d'estimatologia, és a dir, sobre la puntuació i els signes de puntuació, influït en part pels estudis de Martin Hubert, O.P., (publicats a començaments dels anys 70 a Archivum Latinitatis Medii Aevi). ${ }^{30}$ L'any 1975 Anglada publicà el seu estudi sobre aquest manuscrit vaticà ${ }^{31}$ i va dirigir diverses memòries de llicenciatura sobre aquest tema, en particular, les presentades per Maria Joana Danés Ribas i Montserrat Guàrdia Rúbies, titulades Stigmatologica (amb el numeral I i II, respectivament), ${ }^{32}$ i la de Jaume Juan que prenia en consideració la puntuació del ms. Lat. 6332 de la BnF. ${ }^{33}$

Fora de Pacià, Anglada rebé l'encàrrec de traduir al castellà el poema Phoenix, erròniament atribuït a Lactanci, per a la collecció Erasmo de textos bilingües de l'Editorial Bosch. ${ }^{34}$ Tanmateix, el seu interès per altres autors de la llatinitat clàssica $\mathrm{i}$ cristiana -com Virgili o Horaci- traspua en altres contribucions, ${ }^{35}$ i sobretot en les memòries de llicenciatura i tesis doctorals que va dirigir a les tres universitats en què va exercir el seu mestratge. ${ }^{36}$ De tots aquests treballs destacaria, seguint un ordre cronològic, la memòria de llicenciatura (UB, 1971), sobre l'estructura del llibre IV de l'Eneida de Virgili, i la tesi doctoral (UAB, 1973), sobre l'art de la composició en Horaci, presentades per Joan Salvadó, ${ }^{37}$ la tesina de llicenciatura de Mariàngela Vilallonga (UAB, 1974), dedicada a analitzar l'estructura “omfàlica" de l'Epistula ad Pisones d'Horaci, ${ }^{38} \mathrm{o}$ la tesi doctoral de Maria Glòria Bages sobre la tradició manuscrita del De ira Dei de

${ }^{27}$ Anglada (2012b).

${ }^{28}$ Anglada (1983a).

${ }^{29}$ En català comptem amb una traducció parcial de dos dels textos pacianencs, l'Exhortació a la penitència i el Tractat sobre el baptisme, a càrrec de Jaume Fàbregas (Fàbregas 1981) que se suma a la vetusta versió de Llorenç Riber (Riber, D’OLot 1931).

${ }^{30}$ Hubert (1969/1970); Hubert (1974).

${ }^{31}$ Anglada (1975).

32 Danés Ribas (1975); Guàrdia Rúbies (1975).

${ }^{33}$ Juan (1975). Tot i no ser-ne el director, em consta que va ser de gran ajuda en l'elaboració de la tesi doctoral del Dr. Juan (Juan 1980), defensada l'any 1980 amb Anglada com a membre del tribunal.

${ }^{34}$ Anglada (1983c). A banda d'aquest poema, la traducció incorporava textos d'altres autors (Claudià, Ovidi, Pomponi Mela, Plini) sobre aquest particular ésser mitològic.

${ }^{35}$ Anglada (1989c).

36 Vegeu també Pérez de Palomar (1975); Rafart (1978).

${ }^{37}$ Salvadó (1971); Salvadó (1973). Per qüestions administratives, qui figura com a director en la tesi doctoral de Salvadó és Virgilio Bejarano.

38 Vilallonga (1974). 
Lactanci, ${ }^{39}$ defensada l'any 1988 a la delegació de Tarragona de la UB -avui Universitat Rovira i Virgili.

La seva formació eclesiàstica i la seva dedicació a la literatura cristiana el feren col-laborar en altres projectes, en particular, en el fallit Corpus Patristicum Hispanum, ${ }^{40}$ de la Fundación Universitaria Española, del qual eixiren només cinc volums, quatre d'ells a càrrec d'Ursicino Domínguez del Val, O.S.A., (), impulsor de la iniciativa. ${ }^{41}$ Per a aquesta colllecció, Anglada, en col-laboració amb Francesc Fonts Monjo, a qui havia dirigit la memòria de llicenciatura (UAB, 1974), ${ }^{42}$ s'havia d'encarregar de l'edició crítica de l'obra de Sever de Menorca.

Per la seva longevitat, Anglada s'ha de comptar entre els darrers representants d'un segment social de tarannà conservador, amarat de cultura eclesiàstica i engendrador d'una rica tradició d'estudis, de notable solidesa formativa, que tingué un protagonisme prou notable en els assoliments de la Filologia Clàssica catalana durant bona part de la vintena centúria. Per orígens familiars i per formació, Anglada pertanyé plenament a aquest milieu social i a la tradició que se'n deriva, però, al mateix temps, les seves estades a Alemanya i el seu accés a les novetats bibliogràfiques europees li feren superar les estretors i els condicionants que els temps imposaven. Tot i que les fornades actuals tendeixen a considerar els esdeveniments del segle XX com quelcom pertanyent a un passat emboirat en la història més remota, el cert és que només dues generacions ens separen dels moments de màxima plenitud d'aquesta tradició de la qual Anglada va heretar l'ofici i la convicció. ${ }^{43}$

\section{ABREVIATURES}

BnF Bibliothèque nationale de France.

INEM Institut Nacional d'Ensenyament Mitjà.

SEEC Sociedad Española de Estudios Clásicos.

UAB Universitat Autònoma de Barcelona.

UB Universitat de Barcelona.

\footnotetext{
${ }^{39}$ Bages (1988).
}

${ }^{40}$ Domínguez del Val (1975), 408-409. En aquesta mateixa col-lecció, Carles Prats, a qui Anglada havia dirigit la memòria de llicenciatura sobre Licinià de Cartagena (Prats Carasa 1970), s'havia d'encarregar de l'edició de l'obra d'aquest mateix autor.

${ }^{41}$ Es tracta dels volums 1 (Leandre de Sevilla) (1979), a càrrec de Jaime Velázquez i 2-5, a càrrec de U. Domínguez del Val que inclouen: 2 (Estudios sobre literatura latina hispano-cristiana) (1986); 3 (Gregori d'Elvira) (1989); 4 (Martín de Braga) (1990); 5 (Historia de la antigua literatura latina hispano-cristiana) (1998).

${ }^{42}$ Fonts Monjo (1974). Anglada també dirigí la tesi doctoral de Pere Brosa Rocabert sobre el novacianisme. Vegeu Brosa Rocabert (1987).

${ }^{43}$ Em complau d'agrair als col-legues Esperança Borrell, Jaume Juan i Noemí Moncunill l'haver volgut llegir i corregir aquesta nota i d'haver-la enriquit amb les seves observacions. Els agraïments s'estenen a Jaime Siles, Director del Departament de Filologia Clàssica, i a Ferran Grau i Ricardo Hernàndez de l'Estudi General - Universitat de València. Per últim, expresso la meva gratitud envers Joan Francesc Alcina, sempre generós i disponible. 


\section{BIBLIOGRAFIA}

AngladA Anfruns, Àngel (1964a). Pacianea. Estudios sobre fuentes de Paciano. Tesis doctoral ... dirigida por Lisardo Rubio, Barcelona, Universitat de Barcelona, 1964.

ANGLADA ANFRUNS, Àngel (1964b). "'Christiano mini nomen est, catholico vero cognomen' a la luz de la doctrina gramatical”, Emerita 32/2, 1964, p. 243-266.

ANGLADA ANFRUNS, Àngel (1965). "La fuente del catálogo heresiológico de Paciano", Emerita 33/2, 1965, p. 321-346.

ANGLADA ANFRUNS, Àngel (1967). “La tradición manuscrita de Paciano de Barcelona", Emerita 35/1, 1967, p. 137-161.

ANGLADA ANFRUNS, Àngel (1975). “La puntuación del ms. Reginensis 331 en el texto de Paciano de Barcelona", Vetera Christianorum 12/2, 1975, p. 269-316.

ANGLADA ANFRUNS, Àngel (1977). “Le corruttele del membro 'aquarum in penitis ignibus fuerit' alla luce della metafora della febbre (Paciano, Paen. 11, 5)", Vetera Christianorum 14, 1977, p. 253-272.

ANGLADA ANFrunS, Àngel (1979). "Unas notas críticas al texto de Paciano de Barcelona", Emerita 47/1, 1979, p. 11-34.

ANGLADA ANFRUNS, Àngel (1980a). “La expresión 'intemerata Dei Virgo' como metáfora del período gramatical en Paciano de Barcelona (Ep. I 3, 1)", Emerita 48/2, 1980, p. 271-294.

ANGLADA ANFrUNS, Àngel (1980b). “Una nota crítica al text de Pacià de Barcelona (Ep. II, 5, 1)", Estudis Universitaris Catalans 24, 1980 (= Miscel-lània Aramon i Serra), p. 5-10.

ANGLADA ANFRUNS, Àngel (1982). Las obras de Paciano publicadas por V. Noguera y edición crítica del Liber de Paenitentibus. Discurso leído en la solemne apertura del curso 198283, València, Universitat de València, 1982.

ANGladA ANFruns, Àngel (1983a). “Notes per a una traducció catalana del De Paenitentibus de Pacià de Barcelona", Faventia 5/1, 1983, p. 89-92.

ANGladA ANFruns, Àngel (1983b). "Otra vez 'aqua cum in penitis ignibus feruerit, supplicio nutriente reparabitur' (Paciano Paen. XI, 5)”, Faventia 5/2, 1983, p. 83-90.

ANGLADA ANFRUNS, Àngel (1983c). El mito del Ave Fénix. Introducción, textos, traducción y notas de Ángel Anglada Anfruns, Barcelona, Editorial Bosch, 1983 (Col. Erasmo. Textos bilingues).

ANGLADA ANFrUnS, Àngel (1983d). “Unes suposades cites de Virgili en Pacià”, Studia Virgiliana. Actes del VIè Simposi d'Estudis Clàssics, 11-13 de febrer de 1981, Bellaterra, Universitat Autònoma de Barcelona, 1985, p. 265-268.

AngladA ANFrunS, Àngel (1984a). “Unas notas críticas del texto de Paciano de Barcelona (Paen. I, 2; III, 2; Ep. II, II, 3; III, 3; IV, 4; V, 1; VII, 1)", Athlon. Satura grammatica in honorem Francisci R. Adrados, coord. por Luis Alberto de Cuenca, Elvira Gangutia Elícegui, Alberto Bernabé Pajares, Javier López Facal, vol. II, Madrid 1984, p. 23-28.

ANGLADA ANFruns, Àngel (1984b). "La editio princeps de las obras de Paciano de Barcelona", Estudis en memòria del professor Manuel Sanchis Guarner. Estudis de llengua $i$ literatura, vol. II, València, Universitat de València, 1984, p. 17-22.

Anglada Anfruns, Àngel (1985). “'Eneida' VI, 595-600 i Pacià 'De Paenitentibus' XI 5, 311-316", Els gèneres literaris. Actes del VII Simposi d'Estudis Clàssics, 21-24 de Març de 1983, Bellaterra, Universitat Autònoma de Barcelona, 1985, p. 255-258.

ANGLADA ANFrunS, Àngel (1986a). "Las apostillas de Latino Latini al texto de Paciano de Barcelona", Vetera Christianorum 23, 1986, p. 231-266. 
Anglada Anfruns, Àngel (1986b). "Estudiant amb peu de rei Pacià Paen. V. 3, 121", Arxiu de Textos Catalans Antics 5, 1986, p. 271.

ANGLADA ANFrunS, Àngel (1986c). "El texto de Paciano en la bibliotheca Patrum de Marguerin de la Bigne", Homenaje a Pedro Sáinz Rodríguez. Vol. I. Repertorios, textos y comentarios, Madrid, Fundación Universitaria Española, 1986, p. 309-337.

ANGLADA ANFRUNS, Àngel (1986-1987). “'Ornationis ornatus' en Paciano. La longitud de los miembros del periodo", Cuadernos de Filología Clásica 20, 1986-1987, p. 41-58.

Anglada ANFruns, Àngel (1988a). "Revisando mi edición del De Paenitentibus de Paciano de Barcelona", Faventia 10/1-2, 1988, p. 65-76.

ANGLADA ANFRUNS, Àngel (1988b). “La nota marginal 'Maria ex stirpe Danielis' en laedición de Paciano del cardenal Sáenz de Aguirre”, Emerita 66/1, 1998, p. 23-26.

AngladA ANFruns, Àngel (1989a). "Consideracions sobre el ritmo de la prosa de Paciano", Helmantica 40, 121-123, 1989 [= Actas del I Simposio de latín cristiano, ed. J. Oroz Reta], p. 21-43.

ANGLADA ANFRUNS, Àngel (1989b). “Género literario de la carta segunda de Paciano de Barcelona: clasificación", Actas del VII Congreso Español de Estudios Clásicos (Madrid, 20-24 de abril de 1987), vol. II, Madrid 1989, p. 457-462.

AngladA ANFruns, Àngel (1989c). "Horacio, C. I 7, 27-29”, Actas del VII Congreso Español de Estudios Clásicos (Madrid, 20-24 de abril de 1987), vol. II, Madrid 1989, p. 463468.

AngladA Anfruns, Àngel (1991a). "Los Adversaria de Kaspar von Barth sobre el texto de Paciano", Excerpta philologica: Revista de filología griega y latina de la Universidad de Cádiz 1/1, 1991, p. 35-56.

ANGLADA ANFRUNS, Àngel (1991b). “Unas notas de crítica textual sobre nombres hebreos en Paciano de Barcelona", Emérita 59/1, 1991, p. 155-182.

ANGLADA ANFRUNS, Àngel (1994): "Sobre la dimensio syllabraum en Paciano de Barcelona", Actas del VIII Congreso Español de Estudios Clásicos, Madrid, 1994, vol. I, p. $397-$ 404.

ANGLADA ANFruns, Àngel (1997a). “La fuente de la edición del De Baptismo de Paciano en 'España Sagrada' de E. Flórez", Analecta Malacitana 20/2, 1997, p. 433-445.

ANGLADA ANFrUNS, Àngel (1997b): “Carmelo Granado. Pacien de Barcelone. Écrits", Arxiu de Textos Catalans Antics 16, 1997, p. 483-489.

ANGLADA ANFruns, Àngel (1998). “Pacià en el ms. 1631 de la Bibliothèque Mazarine de París", Arxiu de Textos Catalans Antics 17, 1998, p. 472-489.

ANGLADA ANFrUNS, Àngel (1999). "Comentario sobre la edición reciente de Paciano", Analecta Malacitana 22/1, 1999, p. 305-339.

ANGladA ANFruns, Àngel (2006). “De Paciano in codicibus Lugdunensi 5804 et Vitryatensi 2", Vetera Christianorum 43, 2006, p. 31-52.

ANGlADA ANFrUns, Àngel (2012a). In Paciani episcopi Barcinonensis opera silva studiorum, Turnhout, Brepols, 2012.

ANGLADA ANFrUns, Àngel (2012b). Paciani Barcinonensis opera quae extant, Turnhout. Brepols (Corpus Christianorum. Series Latina 69B), 2012.

BAGES FERRER, Maria Glòria (1988). La tradició manuscrita del tractat 'De ira Dei' de Lactanci. Tesi presentada per a l'obtenció del grau de doctor a la Facultat de Filosofia i Lletres de Tarragona, dirigida per Àngel Anglada Anfruns, Tarragona, Facultat de Filoosfia i Lletres de Tarragona, 1988. 
Bertrand, Dominique, Busquets, Jordi, MAYER, Marc, Pacien de Barcelone et l'Hispanie au IV siècle (Actes des colloques de Barcelone et de Lyon, mars et octobre 1996), Paris, Les Éditions du Cerf - Facultat de Teologia de Catalunya, 2004.

Brosa Rocabert, Pedro (1987): Novatianea. Estudio de las citas bíblicas en las fuentes latinas del novacianismo. Tesis de doctorado que ... presenta Pedro Brosa Rocabert. Realizada bajo la dirección de Ángel Anglada Anfruns, Barcelona, Universitat de Barcelona, 1987.

DANÉS RIBAS, Maria Joana (1975). Stigmatologica I. Memoria de licenciatura de María Juana Danés Ribas. Director: Ángel Anglada Anfruns, Barcelona, Universitat de Barcelona, 1975.

DOMÍNGUEZ DEL VAL, Ursicino (1975). “Una nueva colección patrística: Corpus Patristicum Hispanum", Didaskalia 5, 1975, p. 405-410.

FÀBREGAS I BAQUÉ, Jaume (1981). Sant Pacià de Barcelona. Exhortació a la penitència $i$ Tractat sobre el baptisme. Introducció, versió i notes per Jaume Fàbregas i Baqué, Barcelona, Facultat de Teologia, 1981 (Testimonis litúrgics, 6).

FONTS MONJO, Francisco (1974). Estudio sobre la complosión cuantitativa en el libro primero de la Eneida de Publio Virgilio Marón. Tesis de licenciatura dirigida por el Dr. D. Àngel Anglada Anfruns, Bellaterra, Universitat Autònoma de Barcelona, 1974.

GARCíA GIL, María Ángeles (1960). Sintaxis de los casos en la obra literaria de San Paciano. Tesis de licenciatura, Barcelona, Universitat de Barcelona, 1960.

GRANADO, Carmelo (1995). Pacien de Barcelone. Écrits, Introduction, Texte critique, Commentaire etIndex. Traduction par Chantal Épitalon et Michel Lestienne, París, Editions du Cerf (Sources Chrétiennes, 410), 1995.

GUÀRDIA RÚBIES, Montserrat (1975). Stigmatologica II. Memoria de licenciatura de Montserrat Guardia Rubies. Director: Àngel Anglada Anfruns, Barcelona, Universitat de Barcelona, 1975.

HUBERT, Martin (1969/1970). "Corpus stigmatologicum minus", Archivum Latinitatis Medii Aevi 37, (1969/1970), p. 5-171.

HUBERT, Martin (1974). "Corpus stigmatologicum minus. Index", Archivum Latinitatis Medii Aevi 9, (1974), p. 55-80.

JuAN, Jaume (1975). El Manuscrito de Cicerón parisinus 6332: Cato maior. Transcripción y estudios de puntuación. Memoria de Licenciatura de Jaime Juan Castelló. Director ponente: Ángel Anglada Anfruns, Barcelona, Universitat de Barcelona, 1975.

JUAN, Jaume (1980). La puntuació llatina i la lectura en veu alta. Tesis doctoral dirigida per J.I. Ciruelo Borge, Barcelona, Universitat de Barcelona, 1980.

Oliveras CAMINAL, Josep (1949). El problema de la divisibilidad del continuo, con el apendice codicum in Sanctae Barcinonensis Ecclesiae segregatis asservatorum tabulae. Oración inaugural del Curso Academico 1949-1950, Barcelona, Seminari Conciliar de Barcelona, 1949.

Oliveras CAMINAL, Josep (1952). “Codicum in sanctae Barcinonensis ecclesiae segregatis asservatorum tabulae", Scrinium 7, 1952, p. 6-16.

PéREZ De PAlOMAR y MeNDiguRen, Fernando (1975). Religión y cristianismo en Roma. Memoria de licenciatura de Fernando Pérez de Palomar y Mendiguren. Director: Ángel Anglada, Barcelona, Universitat de Barcelona, 1975.

PRATS CARASA, Carles (1970). Estudios sobre el ritmo de la prosa en Liciniano de Cartagena. Memoria de licenciatura de Carlos Prats Carasa, bajo la dirección del Prof. Dr. D. Àngel Anglada Anfruns, Barcelona, Universitat de Barcelona, 1970. 
RAFART CASEllas, Raimon (1978). Estudios sobre el De bono pudicitiae. Memoria de licenciatura de Raimon Rafart Casellas. Director: Ángel Anglada Anfruns, Barcelona, Universitat de Barcelona, 1978.

Requena Gallego, María Dolores (1960). Conjunciones de subordinación en el latín de San Paciano. Memoria de Licenciatura, Barcelona, Universitat de Barcelona, 1960.

RiBer, Llorenç, D'Olot, Xavier (1931). Sant Pacià. Obres. Versió de Mn. Llorenç Riber, prev. Marc Minuci Fèlix. Octavi. Versió del P. Xavier d'Olot, O. M. Cap., Barcelona, Llibreria Barcelona, 1931.

Rubio, Lisardo (1957). “El texto de San Paciano", Emerita 25/2, p. 327-367.

RuBIO, Lisardo (1958). San Paciano. Obras. Edición crítica y traducción, Barcelona, Universitat de Barcelona (Biblioteca de autores barceloneses), 1958.

SALVADÓ, Joan (1971). Correspondencia de patrones en el Libro IV de la Eneida. Memoria de Licenciatura de Juan Salvadó Recasens, bajo la dirección del Prof. Dr.D. Angel Anglada Anfruns, Barcelona, Universitat de Barcelona, 1971.

SALVADÓ, Joan (1974): Latina Symmetrica. Estudios sobre el arte de la composición en Horacio: Odas y Epodos. Tesis doctoral dirigida por el Dr. Virgilio Bejarano, Bellaterra, Universitat Autònoma de Barcelona, 1973.

SENTÍs ANFRUNS, Carlos (2006). Memorias de un espectador (1911-1950), Barcelona, Destino, 2006.

TOVAR PAZ, Francisco Javier (1994). “El género literario de las obras no epistolares de Paciano de Barcelona", Fortunatae. Revista canaria de filología, cultura y humanidades clásicas 6(1994), 315-327.

VILALLONGA, Mariàngela (1974). La técnica repetitiva en la 'Epistola ad Pisones' de Horacio dirigida por el Dr. Angel Anglada, Bellaterra, Universitat Autònoma de Barcelona, 1974. 